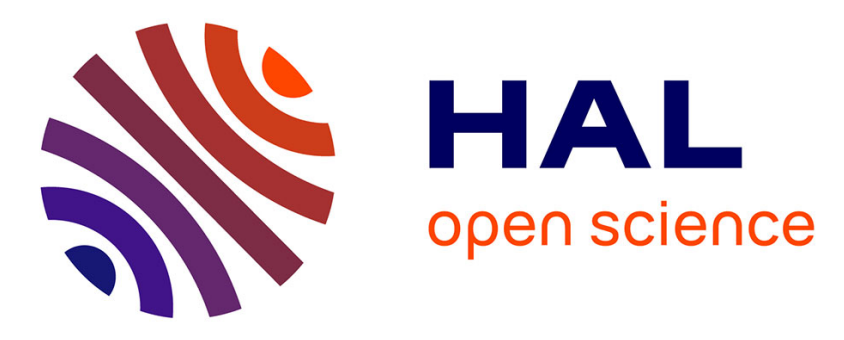

\title{
Numerical Model Reduction for the Prediction of Interface Pressure Applied by Compression Bandages on the Lower Leg
}

Fanette Chassagne, Jérôme Molimard, Reynald Convert, Pascal Giraux, Pierre Badel

\section{To cite this version:}

Fanette Chassagne, Jérôme Molimard, Reynald Convert, Pascal Giraux, Pierre Badel. Numerical Model Reduction for the Prediction of Interface Pressure Applied by Compression Bandages on the Lower Leg. IEEE Transactions on Biomedical Engineering, 2018, 65 (2), pp.449-457. 10.1109/TBME.2017.2774598 . hal-02318360

\author{
HAL Id: hal-02318360 \\ https://hal.science/hal-02318360
}

Submitted on 17 Oct 2019

HAL is a multi-disciplinary open access archive for the deposit and dissemination of scientific research documents, whether they are published or not. The documents may come from teaching and research institutions in France or abroad, or from public or private research centers.
L'archive ouverte pluridisciplinaire HAL, est destinée au dépôt et à la diffusion de documents scientifiques de niveau recherche, publiés ou non, émanant des établissements d'enseignement et de recherche français ou étrangers, des laboratoires publics ou privés. 


\title{
Numerical model reduction for the prediction of interface pressure applied by compression bandages on the lower leg
}

\author{
Fanette Chassagne*, Jérôme Molimard, Reynald Convert, Pascal Giraux, Pierre Badel,
}

\begin{abstract}
Objective: To develop a new method for the prediction of interface pressure applied by medical compression bandages. Methods: A finite element simulation of bandage application was designed, based on patient-specific leg geometries. For personalized interface pressure prediction, a model reduction approach was proposed, which included the parametrization of the leg geometry. Pressure values computed with this reduced model were then confronted to experimental pressure values. Results: The most influencing parameters were found to be the bandage tension, the skin-to-bandage friction coefficient and the leg morphology. Thanks to the model reduction approach, it was possible to compute interface pressure as a linear combination of these parameters. The pressures computed with this reduced model were in agreement with experimental pressure values measured on 66 patients' legs. Conclusion: This methodology helps to predict patient-specific interface pressure applied by compression bandages within a few minutes whereas it would take a few days for the numerical simulation. The results of this method show less bias than Laplace's Law, which is for now the only other method for interface pressure computation.
\end{abstract}

Index Terms-Finite element simulation; model reduction; compression bandages; interface pressure

\section{INTRODUCTION}

Compression bandages consist in stretched fabrics wrapped around the lower limb. This therapy, whose efficacy is admitted [1], is usually used in the treatment of venous or lymphatic pathologies. The stretched fabric applies a pressure onto the skin, called interface pressure, which is then transmitted to the vessels through soft tissues (mainly adipose and muscle tissues) [2]. The target interface pressure, which is a key aspect of the treatment [3], may vary with the pathology to be treated. Indeed, the pressure required for severe pathologies such as venous leg ulcers may be over $40 \mathrm{mmHg}$ [4] whereas other pathologies would require lower interface pressures [5]. More than the success of the treatment, this target pressure can also impact the treatment observance [6], hence an important need to control it. Interface pressure generated by compression bandages results from complex phenomena [7] [8] which remain poorly understood [9]. On the one hand, this pressure is impacted by the fabric material:

F. Chassagne is with the Ecole Nationale Supérieure des Mines de Saint-Etienne, CIS-EMSE, SAINBIOSE, Saint-Etienne, France and Thuasne, BP243, 92307 Levallois-Perret cedex, France (email: fanette.chassagne@emse.fr).

J. Molimard and P. Badel are with the Ecole Nationale Supérieure des Mines de Saint-Etienne, CIS-EMSE, SAINBIOSE, Saint-Etienne, France.

P. Giraux is with the Department of Physical Medicine and Rehabilitation, Faculty of Medicine, University Jean Monnet, Saint-Etienne, France.

R. Convert is with Thuasne, BP243, 92307 Levallois-Perret cedex, France. its mechanical properties (stiffness characterizing the tension needed to stretch the bandage), its stretch once applied on the leg, the application technique (spiral pattern, figure-ofeight, ...) and the interaction between the bandage layers (i.e. the frictional properties of the fabric) [10]. On the other hand, the pressure varies with the lower leg morphology and its mechanical properties, both dependent on body position. Eventually, Also once applied on the leg, the bandage interacts with the skin through friction phenomena. Finally, interface pressure tends to vary over time because of the viscoelastic behavior of both bandage fabric materials [11] and leg soft tissues [12].

Among the large diversity of commercially available bandages [3], practitioners have to select the one best suited to the patient's morphology and pathology. However, for now, the only method to compute interface pressure is Laplace's law, which states that interface pressure $P$ is directly proportional to the bandage tension $T[\mathrm{~N} / \mathrm{mm}]$ and the number of layers $n$ but inversely proportional to the local radius of curvature $r_{C}$ :

$$
P=\frac{n T}{r_{C}}, T>0, r_{C}>0
$$

Though it was adapted to compression bandage therapy [13] [14], the relevance of the use of this law was called into question [15]. First, the measure of interface pressure applied on 30 healthy subjects' legs by two bandages with similar fabric structure but different tension showed that pressure was not directly proportional to bandage tension $T$ [16]. Moreover, the use of this Law often considers a cylindrical approximation of the leg geometry (considering only the leg circumference) and an homogeneous bandage tension [17]. Eventually, this law does not take into account all parameters impacting interface pressure, like soft tissue deformation induced by bandage application for instance [18].

Another mean to predict interface pressure is finite element simulation. This method was shown to be efficient for the computation of pressure applied by compression stockings, with 2D [19] or 3D models [20], but was rarely implemented to compute pressure applied by compression bandages [18]

Therefore, a new method for fast interface pressure computation, based on a finite element modelling, is proposed in this study. A 3D simulation of bandage application, based on a personalized leg geometry, was designed. Then a model reduction technique was developed, including a novel global geometrical description of the lower leg, to predict 
personalized pressure values, which were then confronted to experimental pressure values.

\section{MATERIALS}

\section{A. Leg geometries}

Two sets of leg geometries were required for this study: a first one for the geometrical parametrization of the leg and a second one for the validation of the reduced model.

The first set of leg geometries was composed of the external geometries of the right leg of 35 subjects (20 women - 15 men; mean age: 43 [25 -58]; mean BMI: 24.1 [18.3 - 33.5]) which took part in two previous studies [16] [18]. These geometries were acquired thanks to a 3D optical scanner (Artec ${ }^{\mathrm{tm}}$ MHT 3D scanner, Artec ${ }^{\mathrm{tm}}$ Group), in standing position. The acquisition method was described in previous articles [16] [18]. These 35 geometries were then used for the parametrization of the leg geometry, detailed in the Methods section (III-A). The second set of geometries was obtained from a more recent study [21], in which both legs of 25 patients (15 women - 10 men; mean age: 48 [19 -72]; BMI: 25.6 [19.0 - 37.8]) were scanned in sitting and standing position, following a similar protocol. Among these scans, 38 represented the complete lower leg and thus were suitable for the validation of the reduced model detailed in the Methods section (III-D).

\section{B. Interface pressure measurements}

In addition to leg shape measurements, interface pressures applied by different bandages were measured in the three previously cited studies [16] [18] [21].

First, two different elastic bandages (Biflex ${ }^{\circledR} 16$ and Biflex ${ }^{\circledR}$ 17, Thuasne) were applied on the leg of the 35 subjects, whose leg geometries were used for the parametrization.

For the most recent study [21], only the pressure applied on the patients' leg by the Biflex ${ }^{\circledR} 16$ (Thuasne) was considered for the validation of the reduced model.

For both groups, pressure values were measured at measurement point B1 (where the Achille's tendon turns into the gastroscnemius muscles [22]) thanks to pressure sensor Picopress ${ }^{\circledR}$ (Microlab, Padua, Italia). The measurement protocol was described in previous articles [16] [18] [21]. These measurements were then compared with the results given by the reduced model in the Results section (IV-B).

\section{METHODS}

\section{A. Geometrical parametrization of the lower leg}

To compute interface pressure with Laplace's law, the leg is geometrically described by its local radius of curvature. For subsequent model reduction, another parametrization of the global leg geometry was needed in the present study. This parametrization should require as few parameters as possible for the leg description within an acceptable reconstruction error. This objective was reached thanks to the Proper Orthogonal Decomposition (POD) of the 35 leg geometries previously described in the Materials section (II-A).

These geometries were obtained from the 3D optical seans of the right leg (in standing position) of 35 subjects ( 20 women-
15 men) from previous studies . They These geometries were all set in the same reference frame, then normalized to their lengths and divided into 100 sections (in the transverse plane). Each section was then approximated with Fourier polynomials:

$$
r(\theta, z)=r_{0}+\sum_{m=1}^{n} a_{m} * \cos (m \theta)+\sum_{m=1}^{n} b_{m} * \sin (m \theta)
$$

with $n=5$ the degree of the polynomials and $r(\theta, z)$ the radius for a given angle $\theta$ and a given height $z$ (cylindrical coordinates system). For $n=5$, each section was described with 11 Fourier polynomials coefficients and the coordinates of the centre $\left(x_{0}, y_{0}\right)$, so 13 parameters. Thereby, each leg geometry $\left\{U^{i}\right\}_{i=1 . . N}$, previously normalized to its length, was described with 1300 parameters (100 sections described by 13 parameters). The same methodology as described by Gogu et al. [23] was followed to build the POD basis. All computations were implemented with Matlab ${ }^{\circledR}$ (Mathworks, Natick, MA, USA). The $N=35$ leg geometries could then be approximated as a linear combination of the vectors of the orthogonal basis:

$$
\tilde{U}^{i}=\sum_{k=1}^{K} \alpha_{i, k} \phi_{k}=\sum_{k=1}^{K}<U^{i}, \phi_{k}>\phi_{k}, \quad K \leq N
$$

with $\tilde{U}^{i}$ the approximated leg geometry, $\phi_{k}$ the vectors of the orthogonal POD basis, $K$ the size of the basis and $N$ the number of leg geometries $U^{i}$. For each section of the $N=35$ leg geometries, an evaluation of the reconstruction error was given by the distance between a point on the approximated section and one on the actual section for a given angle $\theta$ (i.e. $r(\theta, z)-\tilde{r}(\theta, z)$ with $r(\theta, z)$ the actual radius and $\tilde{r}(\theta, z)$ the approximated radius). The median reconstruction error $\left(\begin{array}{ll}U^{i} & \left.\tilde{U}^{i} \|\right)\end{array}\right)$ with regards to the basis dimension $\mathrm{K}$ is presented in Figure 1. With the first 4 POD vectors, 95\% of the reconstruction errors of all measuring points of the 35 legs were found to be smaller than $2 \mathrm{~mm}$ (Figure 1 - A).

\section{B. Numerical Simulation}

A finite element simulation of bandage application was designed with Abaqus ${ }^{\circledR}$. This simulation was based on a 3D lower leg geometry on which a $10-\mathrm{cm}$ wide stretched bandage was wrapped in a spiral pattern. The output of the simulation was the interface pressure measured over a $50 \mathrm{~mm}$ diameter disk located at measurement point B1 on the medial side of the leg (corresponding the Picopress ${ }^{\circledR}$ sensor area (Microlab, Padua, Italia), used in the experimental measurements [16] [21]) (Figure 5 - A).

\section{Leg geometry and mechanical properties}

The external leg geometry was given thanks teconstructed from the geometrical parametrization previously described and was then converted to a 3D volume. The geometry of bone holes (tibia and fibula) was obtained from anatomical slices of the 'Visible Human Server' (Computer Science Department, Peripheral Systems Lab., Ecole Polytechnique Fédérale de Lausanne), then scaled to the 3D leg geometry and implanted 

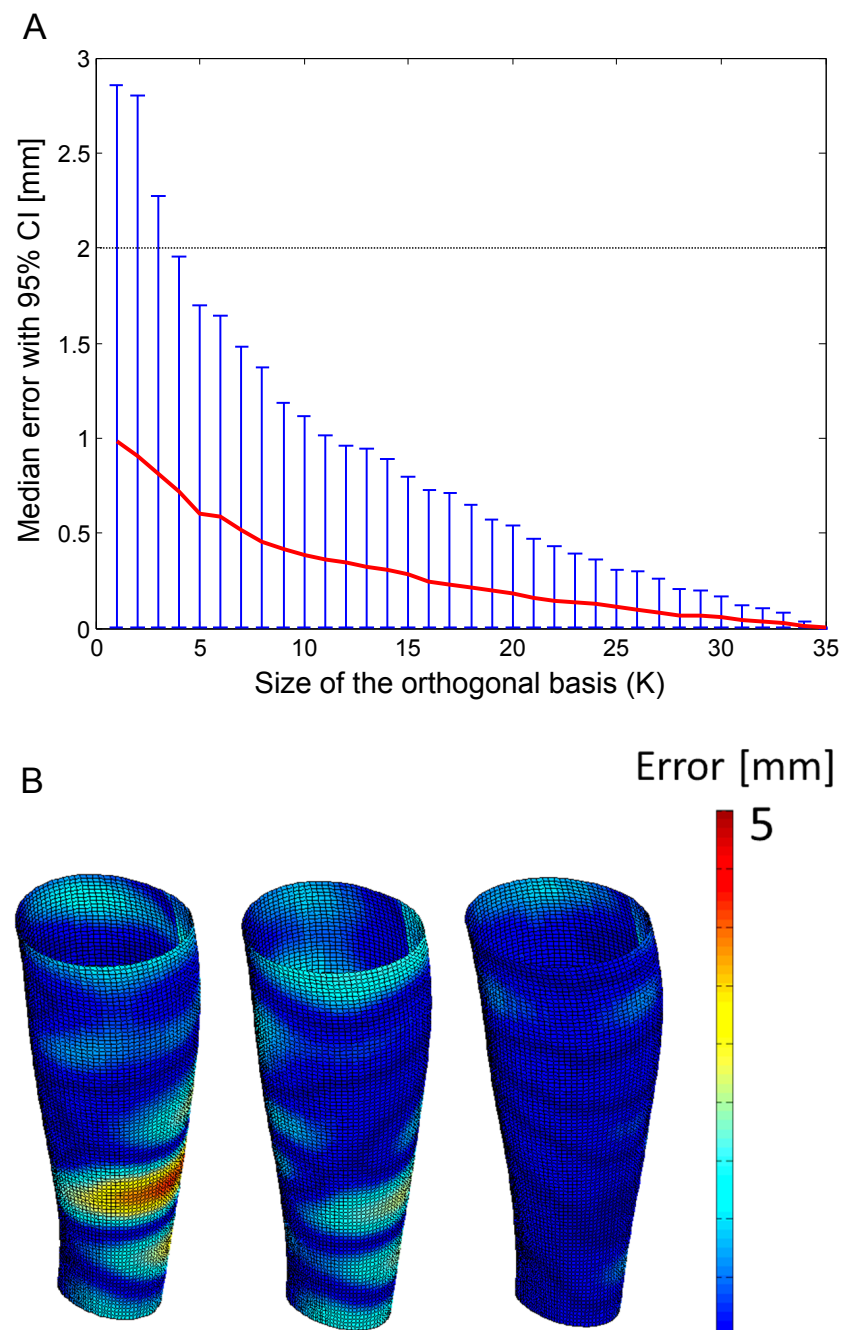

$\mathrm{K}=1$

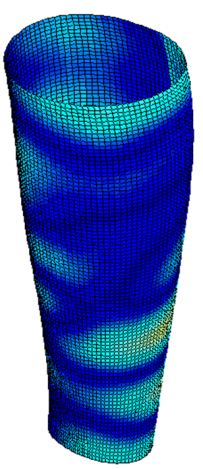

$\mathrm{K}=4$

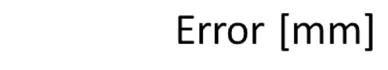

5

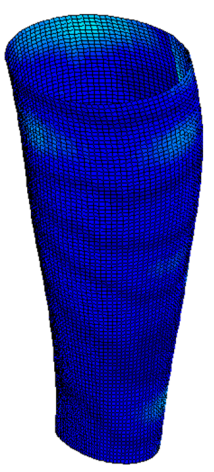

$K=8$
A - Leg geometry given by the parameterization
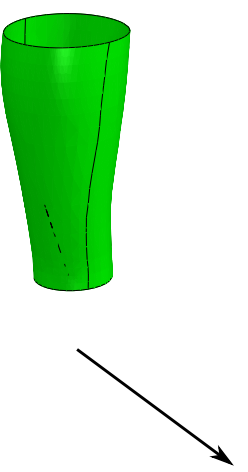

B - Bones geometry (Visible Human Server)
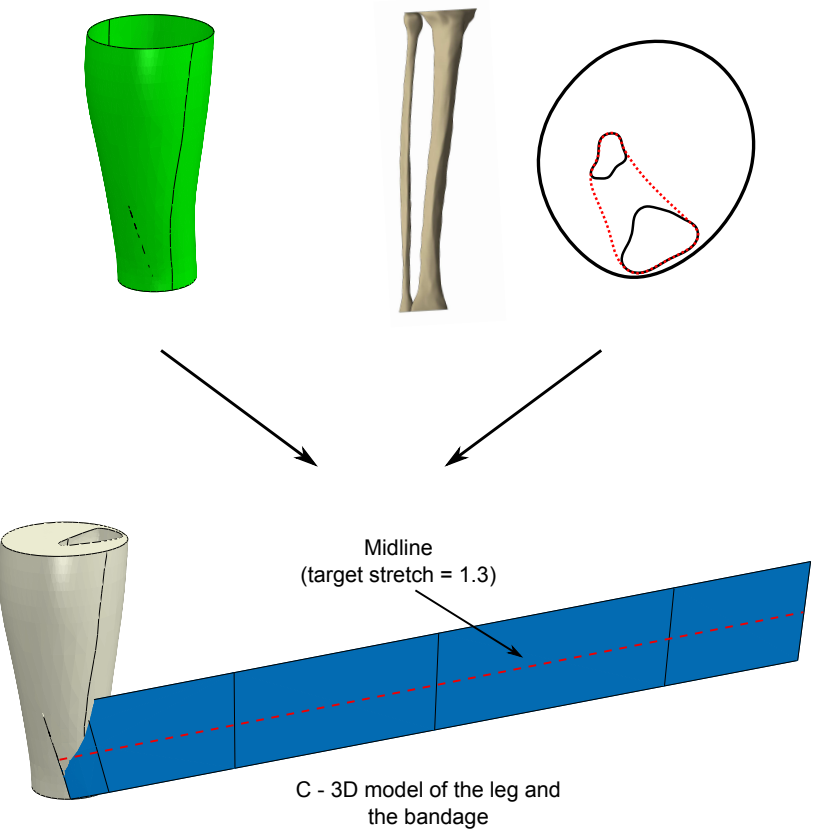

Figure 2: Design of the 3D model: first the leg external geometry is given by the geometrical parametrization (A) and converted to a 3D volume in which bones are implanted (B); eventually, a flat bandage is added to the model (C)

the elastic volume ratio, $c_{10}$ the shear modulus and $\kappa$ the bulk modulus, whose value was taken from the literature $(\kappa=14.3 \mathrm{MPa}[24])$.

\section{Bandage geometry and mechanical properties}

Compression bandage was modelled as a $10-\mathrm{cm}$ wide shell geometry, whose length was adapted to the leg length (Figure 2 - C). It was meshed with 4-node shell elements with reduced integration (about 40,000 elements, depending on the leg geometry).

Compression bandages material was described as an orthotropic linear elastic material. All material parameters were linked by the following equations:

$$
\begin{aligned}
& {\left[\begin{array}{l}
\epsilon_{11} \\
\epsilon_{22} \\
\gamma_{12}
\end{array}\right]=\left[\begin{array}{ccc}
\frac{1}{K_{1}} & -\frac{\nu_{12}}{K_{1}} & 0 \\
\frac{1}{K_{2}} & -\frac{\nu_{12}}{K_{2}} & 0 \\
0 & 0 & \frac{1}{G}
\end{array}\right]\left[\begin{array}{l}
N_{11} \\
N_{22} \\
N_{12}
\end{array}\right]} \\
& {\left[\begin{array}{l}
M_{11} \\
M_{22} \\
M_{12}
\end{array}\right]=\left[\begin{array}{ccc}
F_{1} & \tilde{0} & 0 \\
\tilde{0} & F_{2} & 0 \\
0 & 0 & \tau_{12}
\end{array}\right]\left[\begin{array}{l}
\kappa_{11} \\
\kappa_{22} \\
\kappa_{12}
\end{array}\right]}
\end{aligned}
$$

with $\epsilon_{11}$ and $\epsilon_{22}$ the strains in the in-plane longitudinal and

with $\bar{I}_{1}$ the first invariant of the isochoric deformation, $J^{e l}$ 


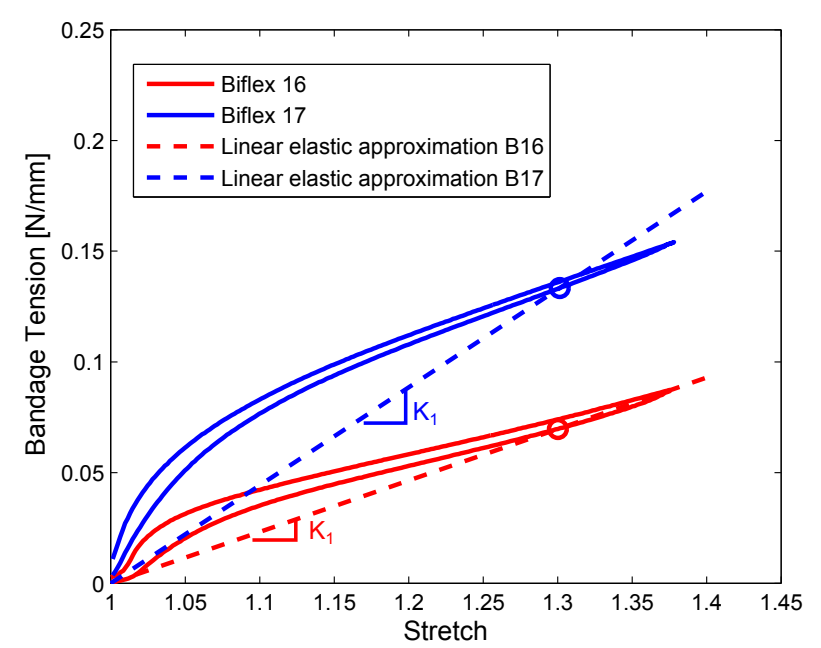

Figure 3: Illustration of two secant elastic moduli from two tension-stretch curves

transverse directions (i.e. the warp and weft directions), $N_{11}$ and $N_{22}$ the section forces in the in-plane longitudinal and transverse directions, $\gamma_{12}$ and $N_{12}$ the in-plane shear strain and section force, $K_{1}$ and $K_{2}$ the elastic moduli in the in-plane directions, $\nu_{12}$ the Poisson's ratio and $G$ the shear modulus, $M_{11}, M_{22}$ and $M_{12}$ the section moments and $\kappa_{11}$, $\kappa_{22}$ and $\kappa_{12}$ the bending strains.

The material parameter of interest in the study was the elastic modulus in the longitudinal direction $K_{1}$. The other parameters $K_{2}, \nu_{12}, G, F_{1}, F_{2}$ and $\tau_{12}$ were set to the values measured in a previous study [18].

The elastic modulus in the longitudinal direction $K_{1}$ was given by the tension of the bandage $T$ at a 1.3 stretch (circle in Figure 3) (secant modulus, ensuring the correct tension value at the prescribed 1.3 stretch).

\section{Simulation strategy}

The edge of the bones hole was pinned. The bandage was tied to the leg close to the ankle, then stretched to a 1.3 stretch (following manufacturer's recommendations) and wrapped on the leg in a 50\% overlapping spiral-pattern application technique (Figure 4). The kinematics of bandage application (i.e. wrapping the bandage around the leg) was computed with an in-house Matlab ${ }^{\circledR}$ code for each leg geometry. The aim of these kinematic boundary conditions was to wrap the bandage, in a 2-layer spiral pattern, with a stretch equal to 1.3 at the midline of the bandage (Figure $2-\mathrm{C}$ ), as the operator would do in the experiments. The contact between the leg and the bandage but also the contact between the different bandage layers allowed no penetration and were modeled with the Coulomb's friction law. Depending on the size of the leg, computation time varied from 1 to 3 days on 12 CPUs.

Output of the simulation

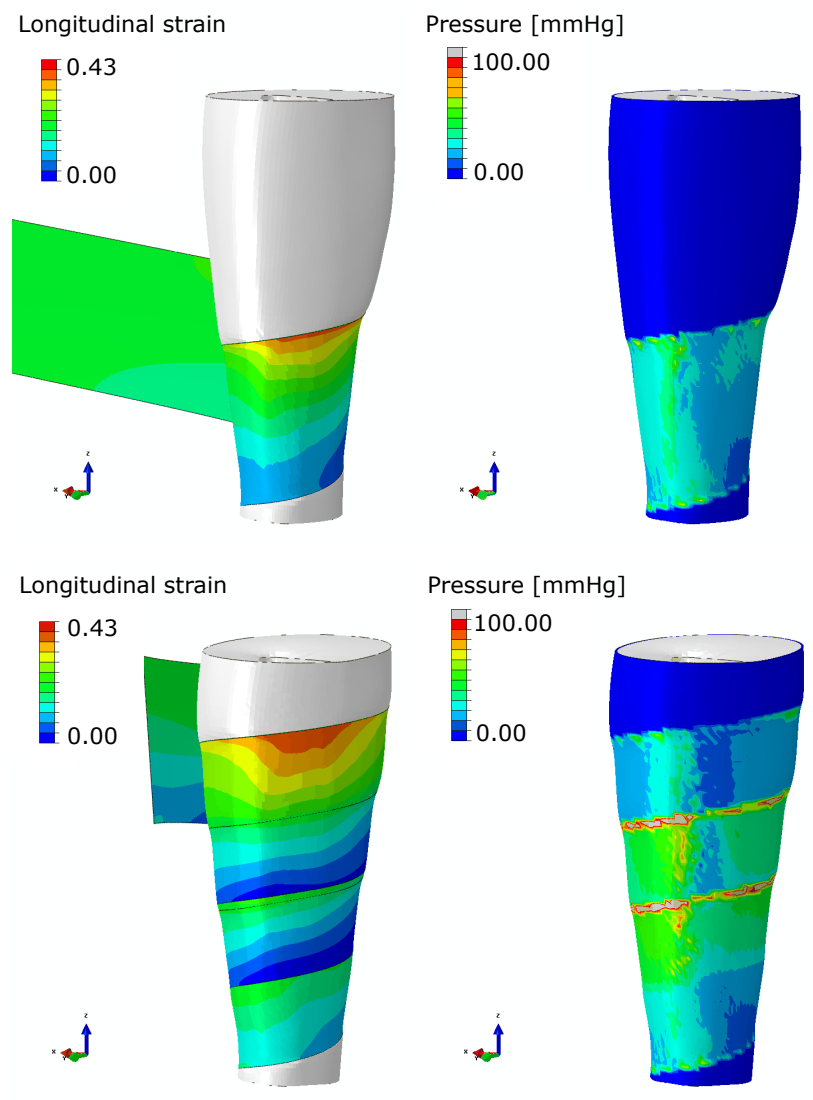

Figure 4: Illustration of the simulation - bandage application on the leg and resulting interface pressure distribution (top: first turn, bottom: end of simulation)

Within the aim later compareing the values from the simulation with the experimental pressure values, the output of the simulation was the mean pressure over an area corresponding to the sensor Picopress ${ }^{\circledR}$ located at measurement point B1 (Figure 5 - A). This area of interest was determined from the curvature of the posterior part of the leg (in blue in Figure 5). Starting from the top of the zone of interest (the top of the leg was not taken into account), in green in Figure 5 , the height of measurement point B1 was the first point with a curvature derivative lower than $\epsilon$ (very close to 0) (in red in Figure 5). This methodology was used to determine the location of measurement point B1 for all legs.

\section{Numerical model reduction}

From this numerical simulation, a model reduction approach was used to assess the impact of different parameters on interface pressure. This approach helps to model a complex phenomenon, in this case interface pressure at measurement point B1, depending on a large number of parameters, with a very few repetitions of the simulation [25].

The parameters possibly impacting interface pressure were:

- the leg geometry,

- the leg soft tissue mechanical properties $\left(c_{10}\right.$ - (Equation 4))

- the bandage tension $(T)$ 
A

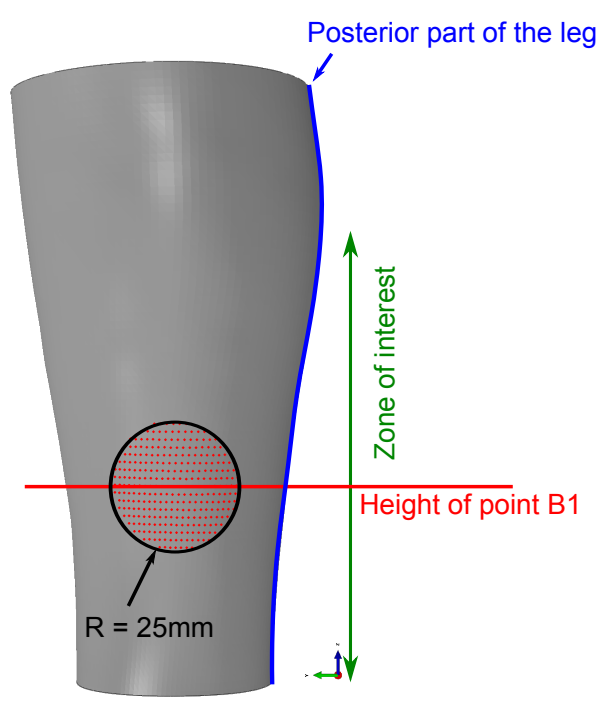

B

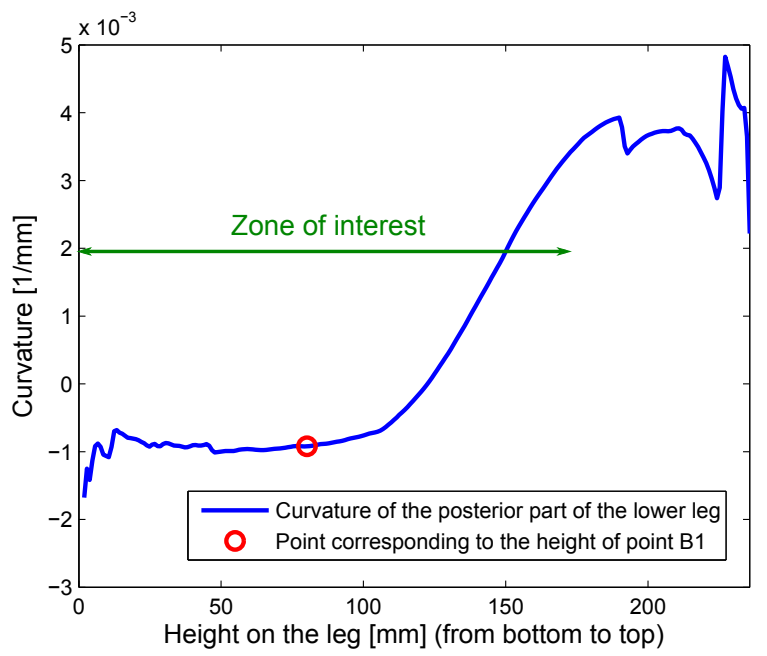

Figure 5: Location of the output of the simulation on the mean leg geometry, i.e. a 50-cm diameter area around measurement point B1 (A); Determination of the height of measurement point $\mathrm{B} 1$ from the curvature of the posterior part of the leg (B)

- the skin-to-bandage friction coefficient $\left(\mu_{\text {skin-bandage }}\right)$

- the bandage-to-bandage friction coefficient $\left(\mu_{\text {bandage-bandage }}\right)$.

First, a study was performed on a mean leg geometry in order to evaluate the impact of the four remaining parameters on interface pressure (soft tissue mechanical properties, bandage tension and the two friction coefficients). The mean leg geometry was obtained by setting all the geometrical parameters to their mean value $\bar{\alpha}_{k}$ (obtained from the 35 geometries used for the parametrization):

$$
U^{\text {mean leg }}=\sum_{k=1}^{35} \bar{\alpha}_{k} \phi_{k}
$$

The impact of the variation of the four parameters was evaluated within the ranges given in Table I. A preliminary

\begin{tabular}{|c|c|c|}
\hline Parameter & $\begin{array}{c}\text { Lowest } \\
\text { value }\end{array}$ & $\begin{array}{c}\text { Highest } \\
\text { value }\end{array}$ \\
\hline $\begin{array}{c}\text { Skin-to-bandage friction } \\
\text { coefficient } \mu_{\text {skin-bandage }}\end{array}$ & 0.10 & 0.30 \\
\hline $\begin{array}{c}\text { Bandage-to-bandage friction } \\
\text { coefficient } \mu_{\text {bandage-bandage }}\end{array}$ & 0.50 & 0.70 \\
\hline Bandage tension $T[\mathrm{~N} / \mathrm{mm}]$ & 0.059 & 0.137 \\
\hline $\begin{array}{c}\text { Leg soft tissue mechanical } \\
\text { properties } c_{10}[\mathrm{kPa}]\end{array}$ & 2.00 & 8.00 \\
\hline
\end{tabular}

Table I: Ranges of variation for the skin-to-bandage friction coefficient $\left(\mu_{\text {skin-bandage }}\right)$, the bandage-to-bandage friction coefficient $\left(\mu_{\text {bandage-bandage }}\right)$, the bandage tension $(T)$ and the soft tissue material parameter $\left(c_{10}\right)$

\begin{tabular}{|c|c|c|}
\hline Parameter & $\begin{array}{c}\text { Lowest } \\
\text { value }\end{array}$ & $\begin{array}{c}\text { Highest } \\
\text { value }\end{array}$ \\
\hline Bandage tension $T[\mathrm{~N} / \mathrm{mm}]$ & 0.059 & 0.137 \\
\hline Lower leg length $L[\mathrm{~mm}]$ & 184.86 & 290.43 \\
\hline $1^{\text {st }}$ geometrical parameter $\alpha_{1}$ & -0.920 & -0.503 \\
\hline $2^{\text {nd }}$ geometrical parameter $\alpha_{2}$ & -0.109 & 0.119 \\
\hline $3^{\text {rd }}$ geometrical parameter $\alpha_{3}$ & -0.092 & 0.068 \\
\hline $4^{\text {th }}$ geometrical parameter $\alpha_{4}$ & -0.081 & 0.095 \\
\hline
\end{tabular}

Table II: Ranges of variation for the skin-to-bandage friction coefficient $\left(\mu_{\text {skin-bandage }}\right)$, the bandage tension $(T)$, the leg length $(L)$ and the four geometrical parameters $\left(\alpha_{1 . .4}\right)$

numerical study was performed to evaluate the variation of interface pressure induced by a variation of skin-to-bandage friction coefficient within the range found in the literature [26] [27]. The lowest value was obtained from the literature $(0.10)$ and the maximum one corresponded to complete adhesion (0.3) (higher values would not lead to lower sliding).

A similar methodology was followed for the range of variation of leg soft tissue mechanical properties, going from 2 to $8 \mathrm{kPa}$ (higher values led to no difference in interface pressure). Minimum and maximum bandage tensions were chosen with regards to the tension of bandages Biflex ${ }^{\circledR} 16$ and Biflex ${ }^{\circledR} 17$ (Figure 3) and the experimental stretches measured in a previous study [16]. The bandage-to-bandage friction coefficient range of variation was given by previous experimental measurements [28].

The evaluation of the impact of these four parameters, in the ranges of variation summarized in Table I, showed that two parameters, skin-to-bandage friction coefficient and bandage tension, were responsible for more than $93 \%$ of interface pressure variation. Consequently, the bandage-to-bandage friction coefficient and the leg soft tissues material parameter were removed from the problem definition and set to their mean values: $\mu_{\text {bandage-bandage }}=0.6$ and $c_{10}=5 \mathrm{kPa}$.

The second step consisted in the evaluation of the impact of skin-to-bandage friction coefficient, bandage tension and leg geometry on interface pressure. The leg geometry was given with 4 geometrical parameters (Equation 3) and the 
leg length $L$. The ranges of variation of the morphological parameters, presented in Table II, were obtained from the 35 leg geometries.

From the ranges of variation defined in Table II, the parameters were coded between -1 and 1 :

$$
X^{*}=2 * \frac{X-X_{\min }}{X_{\max }-X_{\min }}-1
$$

with $X^{*}$ the value of $X$ in the coded space, $X_{\min }$ and $X_{\max }$ the minimum and maximum value of the parameter $X$.

A fractional factorial design was built [25] to select the different combinations of the 7 parameters of interest. Each combination was used to run one simulation and eventually compute the response surface of the model. Here, 9 simulations were run for this model reduction.

From the output of these 9 simulations, i.e. the interface pressure at measurement point $\mathrm{B} 1$ on the medial side of the leg, it was possible to compute this interface pressure $\hat{P}$ at measurement point B1 as a linear combination of skin-tobandage friction coefficient $\mu_{\text {bandage-bandage }}^{*}$, bandage tension $T^{*}$, leg length $L^{*}$ and 4 geometrical parameters $\alpha_{1 . .4}^{*}$ :

$$
\begin{array}{r}
\hat{P}=\bar{P}+\beta_{\mu} \mu_{\text {bandage-bandage }}^{*}+\beta_{T} T^{*}+\beta_{L} L^{*} \\
+\beta_{1} \alpha_{1}^{*}+\beta_{2} \alpha_{2}^{*}+\beta_{3} \alpha_{3}^{*}+\beta_{4} \alpha_{4}^{*}
\end{array}
$$

with all parameters coded between -1 and 1 (Equation 8 ). The coefficients $\beta$ were obtained from the model reduction approach.

D. Comparison between numerical pressure, pressure computed with Laplace's law and experimental pressure values

Experimental pressure measurements applied by two bandages (Biflex ${ }^{\circledR} 16$ and Biflex ${ }^{\circledR}$ 17) were previously performed on the legs of 35 -stbjects, whose geometry was used for the geometrical parametrization [16] [18]. Other pressure measurements applied by the Biflex ${ }^{\circledR} 16$ were performed on both legs of 25 patients [21],whose leg geometry was obtained with a 3D-optical-seanner (Artectm MHT 3D seanner, Artectm Group) in sitting and standing positions.

As it was previously detailed in the Materials section (II), interface pressure measurements were performed on the legs of 35 healthy subjects and 25 patients. These measurements were coupled with leg shape measurements. Among the $3 \mathrm{D}$ scans of the 25 patients' legs in sitting and standing position, 38 represented the complete lower leg and thus were suitable for this study. Once normalized to their length, these 38 leg geometries were decomposed in the orthogonal basis $\left\{\phi_{k}\right\}_{k=1 . .4}$ obtained from the geometrical parametrization. From the 38 sets of geometrical parameters seven were found to be out of the range of variation studied in the model reduction and were discarded.

Eventually, 66 leg geometries and 101 experimental pressure values (two bandages for 35 healthy subjects' legs and one bandage for 31 patients' legs) were kept for the comparison. The pressure applied at measurement point B1 was computed thanks to the linear combination given by the reduced model (Equation 9). As no measurements were performed for the skin-to-bandage friction, this parameter was set to its mean value. In addition, interface pressure applied on the $66 \mathrm{leg}$

\begin{tabular}{|c|c|c|}
\hline $\begin{array}{c}\text { Parameter (coded between -1 } \\
\text { and 1) }\end{array}$ & Coefficient & Value \\
\hline Mean pressure & $\bar{P}$ & 32.73 \\
\hline $\begin{array}{c}\text { Skin-to-bandage friction } \\
\text { coefficient } \mu_{\text {bandage-bandage }}^{*}\end{array}$ & $\beta_{\mu}$ & 2.70 \\
\hline Bandage tension $T^{*}$ & $\beta_{T}$ & 11.54 \\
\hline Lower leg length $L^{*}$ & $\beta_{L}$ & 2.85 \\
\hline $1^{\text {st }}$ geometrical parameter $\alpha_{1}^{*}$ & $\beta_{1}$ & 4.54 \\
\hline $2^{\text {nd }}$ geometrical parameter $\alpha_{2}^{*}$ & $\beta_{2}$ & 3.92 \\
\hline $3^{\text {rd }}$ geometrical parameter $\alpha_{3}^{*}$ & $\beta_{3}$ & -1.74 \\
\hline $4^{\text {th }}$ geometrical parameter $\alpha_{4}^{*}$ & $\beta_{4}$ & -0.51 \\
\hline
\end{tabular}

Table III: Coefficients of the linear model $\beta$ obtained from the model reduction approach (Equation 9)

geometries was computed thanks to Laplace's Law (Equation 1), following the same methodology described in a previous study [18].

For both computations, bandage tension was computed from the experimental stretch measurements:

Tension $=($ stretch -1$) *$ bandage elastic modulus $\left(K_{1}\right)$

Interface pressure values computed with these two methods, reduced model and Laplace's Law, were confronted to experimental values. Note that a previous work showed that the presence of the pressure sensor induced little measurement error, which was previously evaluated thanks to a numerical approach providing a methodology to correct this error [18]. The same methodology was implemented here for the correction of experimental pressure values.

\section{RESUlts}

A new simulation of bandage application was built, within the aim to wrap the bandage around the leg with a 1.3 stretch. The mean stretch measured at the midline of the bandage (Figure 2 - C) was equal to $1.282 \pm 0.001$, but this stretch was very variable over the bandage width: $1.411 \pm 0.004$ at the top edge of the bandage and $1.139 \pm 0.002$ at its bottom edge.

\section{A. Model reduction}

Thanks to the model reduction approach it was possible to compute interface pressure applied at measurement point B1 as a linear combination of seven parameters (Equation 8). The coefficients $\beta$ of the linear combination are reported in Table III.

The overall significance of the linear model obtained from the model reduction was tested with the Fischer test $(p=0.02)$. Moreover, in light of the very high coefficient of determination $R^{2}=0.99$, it can be considered that this seven parameter reduced model gave a relevant description of the variation of interface pressure. 


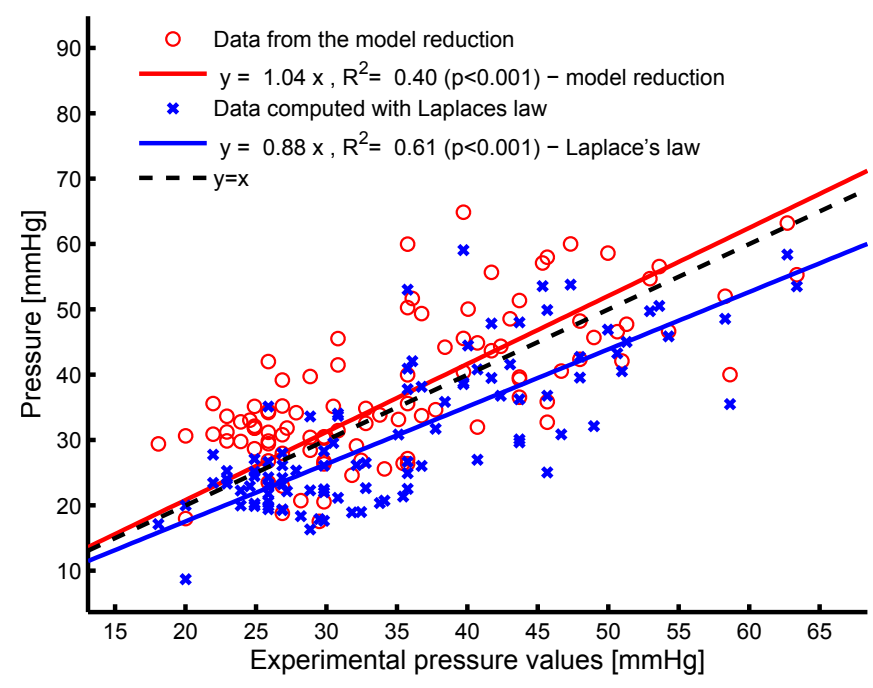

Figure 6: Pressure computed from the model reduction and Laplace's law as a function of the experimental pressure values

\section{B. Comparison of the data computed with the numerical model reduction, Laplace's law and the experimental data}

Pressure was computed from two equations, the reduced model (Equation 9 and Table III) and Laplace's law (Equation 1), for 66 legs, as a function of the experimentally measured bandage stretch. For 35 of them, pressure values applied by two different bandages (Biflex ${ }^{\circledR} 16$ and Biflex ${ }^{\circledR}$ 17) were measured at measurement point B1 [16], whereas for the 31 remaining legs, only the pressure applied by the Biflex ${ }^{\circledR}$ 16 was measured [21]. 101 pressure values, computed from the model reduction equation (Equation 9 and Table III) and Laplace's law (Equation 1), are plotted as a function of the experimental pressure values in Figure 6.

Though the data from the model reduction were scattered, they were in agreement with the experimental data (slope = 1.04 ), with a mean error equal to $9.7 \pm 5.0 \%$. For Laplace's Law, the slope was further to 1 (slope $=0.87$ ) and the mean error was equal to $-11.6 \pm 4.0 \%$. Data from the model reduction were more scattered than the one given by Laplace's law because this model took into account more experimental parameters (seven) than Laplace's Law (two).

\section{DISCUSSION}

A new method, based on the reduction of a numerical simulation, was designed to compute interface pressure applied by compression bandages at measurement point B1.

This method was based on a deformable 3D leg geometry described by 4 geometrical parameters and its length. Other influencing parameters were the bandage tension and the skin-to-bandage friction coefficient. The results obtained from this methodology were in agreement with previous experimental pressure measurements.

The simulation of bandage application was designed to apply the bandage in a spiral pattern with a 1.3 stretch.

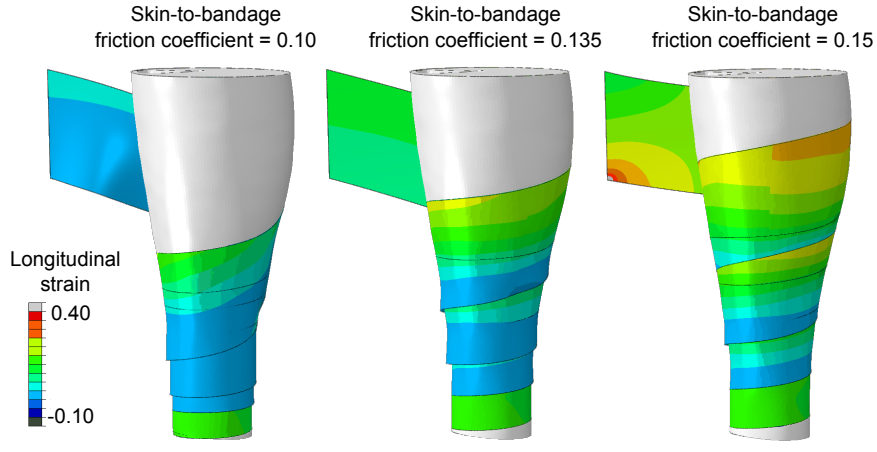

Figure 7: Illustration of the influence of the skin-to-bandage friction coefficient on a very conical leg geometry

The stretch of the numerically applied bandage was found to be slightly lower (1.28). This could be explained by the little compression of the leg soft tissues induced by bandage application and the cross-section becoming rounder. Even though the stretch at the midline was close to the targeted value, it was found to be heterogeneous over the leg (Figure 4 and Figure 7). This stretch heterogeneity was the consequence of the "conical" shape of the leg (stretch is higher in the upper half of the bandage layer and lower in the lower half). Usually, leg geometry is locally described by its radius of curvature. This new global geometrical parametrization, based on 35 right leg geometries, resulted in very low reconstruction error with only 5 parameters (4 geometrical parameters and the leg length) and was suitable to geometrically describe other leg geometries (31 among the 38 available leg geometries). The model reduction approach showed that the last parameters $\alpha_{4}$ had lower effect than the three other geometrical parameters $\alpha_{1 . .3}$ (about $2 \%$ of the mean pressure) (Table III).

The impact of several parameters on interface pressure was evaluated thanks to this model reduction approach. First it was observed that the mechanical properties of soft tissues had not much influence on interface pressure, even though it was shown in a previous numerical study [18] that the deformability of the soft tissue was to be considered to understand pressure generation. Nonetheless, it was previously shown that soft tissue mechanical properties influence the transmission of interface pressure to the vessels [19].

The second parameter with very low impact on interface pressure was bandage-to-bandage friction. The values of this friction coefficient, provided by experimental measurements [28], was very high (from 0.5 to 0.7 ) and did not result in any sliding motion between the different layers, hence its very low impact on pressure. However, bandage layers are in interactions with each other, but also with the skin.

Skin-to-bandage friction coefficient was found to be more influencing than bandage-to-bandage friction in the preliminary study on the mean leg geometry. The values were much lower than for the other frictional parameter. Thus, for the lowest value $(0.10)$ the bandage tended to slide 
down over the leg (Figure 7), whereas for the highest value (0.3), the bandage did not. While sliding down, the stretch of the bandage decreased and so did interface pressure. Nonetheless, as expected considering Coulomb's friction law, it was observed that the impact of skin-to-bandage friction coefficient was influenced by the leg morphology and more especially by the slope of the leg shape. This is the reason why a friction coefficient equal to 0.1 did not lead to much sliding for some leg geometries and led to complete sliding of the bandage for others (Figure 7). This value was in the lowest range of skin-to-textile friction coefficient reported in the literature.

The last parameter impacting interface pressure was, as expected, the tension, which in the study was a function of the bandage elastic modulus and the stretch (Equation 10). However, note that the reduced model is slightly more sensitive to the stretch than reality because the secant elastic modulus is higher than the tangent modulus (Figure 3).

In a second time, the pressure was computed for 66 legs and compared to experimental results. Though predicted pressure values were in agreement with experimental measured values, they sometimes showed large discrepancies. Four possible sources of error were identified.

First, due to the non-cylindrical shape of the leg, wrapping the bandage in a spiral pattern resulted in high pressure areas (in red in Figure 4) induced by the overlapping of the bandage which is not perfectly equal to 2 layers all over the leg (being, sometimes, very locally one or three layers). Whether the sensor is located under such an overlapping zone or not will change the interface pressure. The source of variability was evaluated using the FE model, by moving the center of the area of interest on a $1 \mathrm{~cm}$ radius circle around its initial position and led to a mean absolute pressure variation of $1.27 \pm 0.38 \mathrm{mmHg}$.

The second source of error is the location of the sensor. In the simulation, it was determined from the curvature of the posterior part of the leg (Figure 5). However, experimentally, it is likely that this location slightly differs from the one numerically determined, which may lead to a small pressure variation, of the same order as that evaluated right above.

Also, as it is not possible to patient-specifically characterize the skin-to-bandage friction coefficient, this parameter was set to its mean value. Thus all pressure values computed from the model reduction are given with $\mathrm{a} \pm 2.70 \mathrm{mmHg}$ uncertainty.

The last source of error is body position. Indeed, the bandage is applied in supine position, but it was not possible to scan the leg in this position. So the leg geometries were either obtained in sitting or standing position. These geometries were neither the one on which the bandage was applied, nor the one corresponding to the measured stretch. Due to gravity, muscle groups fall down and bandage stretch may be increased and thus interface pressure may vary.

\section{Limitations}

This methodology was based on the lower leg geometrical parametrization. However, this parametrization was obtained from 35 legs of healthy subjects. Even though these subjects showed a wide range of morphologies, the parametrization should be enriched with more leg geometries, from patients with leg oedema for instance.

For the numerical simulation, the kinematics of bandage application was based on the non-deformed shape of the leg and was not updated during the simulation (to take into account bandage sliding or leg deformation for example). An improvement of the simulation would be to update wrapping boundary conditions during the simulation, after each bandage turn for instance, as an operator does in reality.

The leg geometry was obtained in different body position (sitting or standing) than the position in wich the bandage is applied on the leg (supine position). Therefore it seems crucial to be able to scan the leg geometry in supine position to eliminate the uncertainty about the leg geometry.

Also,-Modelling bandage material as an planar elastic material tended to increase the influence of stretch on interface pressure (secant elastic modulus considered (Figure 3)). Next step would be to consider the non-linear mechanical behaviour of fabric materials in the simulation but also to increase the complexity of the bandage fabric model (by replacing shell elements by solid elements for instance). Another important parameter was the skin-to-bandage friction coefficient. This study did not investigate the coupled effect of the leg geometry and this frictional parameter on interface pressure. Further mechanical studies would be needed for a better understanding of the effect of this frictional parameter.

The output of the simulation was only the pressure value at measurement point B1. However, the complete pressure distribution was provided by the simulation and could be further investigated.

Moreover, the experimental protocol may show some limitations. Bandage application is subject to large variabilities, either for the wrapping pattern (overlapping and angle) or the bandage stretch. Even though all bandages were applied by the same trained operator to prevent inter-operator variability, these sources of uncertainty may also explain the discrepancies between the experiments and the reduced model. Eventually, it seems crucial to be able to scan the leg geometry in the same position in which bandage is applied, to eliminate the uncertainty about the leg geometry. Finally, even if interface pressure was measured within a short time interval to prevent the effect of time, this study did not investigate the viscoelastic behaviour of both bandage materials and leg soft tissues and their impact on interface pressure..

\section{CONCLUSION}

A new method to compute interface pressure applied by compression bandages at measurement point B1 was proposed. This methodology was based on the parametrization of the leg geometry and the numerical simulation of bandage application. In addition to the leg morphology, the bandage tension and the skin-to bandage friction coefficient impacted interface pressure. Thanks to this approach, it is possible to compute patient-specific pressure values in a few minutes 
when it would take a few days with numerical simulation. The pressure values computed for 66 legs were found to be in agreement experimental pressure values. This methodology could later be enriched to compute pressure applied by multi-layer bandages.

\section{REFERENCES}

[1] F. Amsler, T. Willenberg, and W. Blättler. In search of optimal compression therapy for venous leg ulcers: A meta-analysis of studies comparing divers bandages with specifically designed stockings. Journal of Vascular Surgery, 50(3):668-674, September 2009.

[2] B. Partsch and H. Partsch. Calf compression pressure required to achieve venous closure from supine to standing positions. Journal of vascular surgery, 42(4):734-738, October 2005.

[3] Hugo Partsch. Compression for the management of venous leg ulcers: which material do we have? Phlebology, 29, 1 suppl:140-145, May 2014.

[4] S.D. Blair, D.D. Wright, C.M. Backhouse, E. Riddle, and C.N. Mc Collum. Sustained compression and healing of chronic venous ulcers. $B M J$, 297:1159-1161, November 1988.

[5] M. Vicaretti. Compression therapy for venous disease. Australian prescriber, 33:186-90, 2010.

[6] D. J. Milic, S. S. Zivic, D. C. Bogdanovic, M. M. Jovanovic, R. J. Jankovic, Z. D. Milosevic, D. M. Stamenkovic, and M. S. Trenkic. The influence of different sub-bandage pressure values on venous leg ulcers healing when treated with compression therapy. Journal of Vascular Surgery, 51(3):655-661, March 2010.

[7] M. Clark. Compression bandages : principles and definition. European Wound Management Association, pages 5-7, 2003

[8] J. M. Melhuish, M. Clark, R. Williams, and K. G. Harding. The physics of sub-bandage pressure measurement. Journal of wound care, 9(7):308310, July 2000.

[9] H. Partsch. Evidence based compression therapy. VASA, 62 (S/63):1-39, December 2003.

[10] S. Ghosh, A. Mukhopadhyay, M. Sikka, and K.S. Nagla. Pressure mapping and performance of the compression bandage/garment for venous leg ulcer treatment. Journal of Tissue Viability, 17(3):82-94, August 2008.

[11] B. Kumar, A. Das, and R. Alagirusamy. Effect of material and structure of compression bandage on interface pressure variation over time. Phlebology - Venous Forum of the Royal Society of Medicine, April 2013.

[12] Amit Gefen and Einat Haberman. Viscoelastic properties of ovine adipose tissue covering the gluteus muscles. Journal of Biomechanical Engineering, 129(6):924-930, December 2007.

[13] J. Al Khaburi, A. A. Dehghani-Sanij, E. A. Nelson, and J. Hutchinson. Effect of bandage thickness on interface pressure applied by compression bandages. Medical Engineering \& Physics, 34(3):378-385, 2012.

[14] S. Thomas. The use of the Laplace equation in the calculation of subbandage pressure. European Wound Management Association, (3):2123, 2003.

[15] J. Schuren and K. Mohr. The efficacy of Laplace's equation in calculating bandage pressure in venous leg ulcers. WOUNDS UK, 4(2):38-47, 2008.

[16] F. Chassagne, F. Martin, P. Badel, R. Convert, P. Giraux, and J. Molimard. Experimental Investigation of Pressure Applied on the Lower Leg by Elastic Compression Bandage. Annals of Biomedical Engineering, 43(12):2967-2977, December 2015.

[17] R. J. Williams, D. Wertheim, J. Melhuish, and K. G. Harding. How compression therapy works. Journal of Wound Care, 8(6):297-298, June 1999.

[18] F. Chassagne, J. Molimard, R. Convert, P. Giraux, and P. Badel. Numerical Approach for the Assessment of Pressure Generated by Elastic Compression Bandage. Annals of Biomedical Engineering, 44(10):3096-3108, October 2016.

[19] P.-Y. Rohan, P. Badel, B. Lun, D. Rastel, and S. Avril. Prediction of the Biomechanical Effects of Compression Therapy on Deep Veins Using Finite Element Modelling. Annals of Biomedical Engineering, 43(2):314-324, February 2015.

[20] X.Q. Dai, R. Lui, M. Abel, and Y.L. Kwok. Numerical simulation and prediction of skin pressure distribution applied by graduated compression stockings. Computaional textile, 55:301-309, 2007.
[21] F. Chassagne, C. Helouin-Desenne, J. Molimard, R. Convert, P. Badel, and P. Giraux. Superimposition of elastic and non-elastic compression bandages. Journal of Vascular Surgery: Venous and Lymphatic Disorders (in press).

[22] H. Partsch, M. Clark, S. Bassez, J.-P. Benigni, F. Becker, V. Blazek, J. Caprini, A. Cornu-Thénard, J. Hafner, M. Flour, M. Jünger, C. Moffatt, and M. Neumann. Measurement of lower leg compression in vivo: recommendations for the performance of measurements of interface pressure and stiffness: consensus statement. Dermatologic surgery, 32(2):224-232; discussion 233, February 2006.

[23] C. Gogu, W. Yin, R. Haftka, P. Ifju, J. Molimard, R. Le Riche, and A. Vautrin. Bayesian Identification of Elastic Constants in MultiDirectional Laminate from Moiré Interferometry Displacement Fields. Experimental Mechanics, 53(4):635-648, April 2013.

[24] L. Dubuis, S. Avril, J. Debayle, and P. Badel. Identification of the material parameters of soft tissues in the compressed leg. Computer Methods in Biomechanics and Biomedical Engineering, 15(1):3-11, January 2012.

[25] W. Tinsson. Plans d'expérience: constructions et analyses statistiques, volume 67 of Mathématiques et Applications. Springer Berlin Heidelberg, Berlin, Heidelberg, 2010.

[26] L.-C. Gerhardt, A. Lenz, N. D. Spencer, T. Münzer, and S. Derler. Skin-textile friction and skin elasticity in young and aged persons. Skin Research and Technology, 15(3):288-298, August 2009.

[27] L.-C Gerhardt, V Strassle, A Lenz, N.D Spencer, and S Derler. Influence of epidermal hydration on the friction of human skin against textiles. Journal of The Royal Society Interface, 5(28):1317-1328, November 2008.

[28] F. Chassagne, E. Benoist, P. Badel, R. Convert, L. Schacher, and J. Molimard. Characterization of fabric-to-fabric friction: application to medical compression bandages. Textile Research Journal (submitted). 